\title{
Le Métissage Tarentais en Afrique du Nord
}

\author{
$\operatorname{par} M . ' S A I L L A R D$
}

$D_{E}$ nombreuses tentatives d'acclimatement du bétail métropolitain en Afrique du Nord ont été faites; les véritables succès paraissent assez rares pour qu'il nous semble intéressant de faire davantage apprécier les résultats plus qu'encourageants obtenus au Maroc et particulièrement dans le territoire de Zaza, à partir de la race Tarentaise.

En Afrique du Nord, le bétail a généralement peu bénéficié des procédés culturaux et de patientes méthodes zootechniques. Plus pressés d'importer des « sources de lait » que de fournir l'effort de longue haleine, mesuré et difficile, mais productif et raisonnable, beaucoup de producteurs, sans souci pour l'avenir, ont meublé leurs étables de vaches bientôt malades, vite tariç. A côtć d'eux, l'indigèno a traité son cheptel comme tout produit naturel; les disettes périodiques qu'aucune réserve fourragère ne vient atténuer, le sevrage hàtif, ont débilité un bétail exploite sans souci d'amélioration, soumis à une dure existence, n'ayant guère pour lui qu'une rusticité à toute épreuve, une heureuse adaptation au milieu.

Il est des races qu'il sèrait vain de chercher à dépayser. Ce sont celles qui, par une amélioration et une spécialisation trop poussées, ont été étroitemerıl inféodées à un clinrat, à un sol, à un mode particulier d'exploitation. Ce sont toutes celles, enfin, auxquelles leur caractère trop manifestement artificiel ne permet pas la vie des pays chauds. En effet, les races trop précoces, trop éloignées de leur souche, malgré le grand intérêt économique qui s'y attacherait, ne sont pas susceptibles de résister en milieu pénible. C'est, au contraire, vers les races anciennes, rustiques et moins exigeantes, aux qualités d'autant plus solides qu'elles sont originelles, qu'on doit orienter son choix. Un certain nombre de nos vieilles races françaises ont fait leurs preuves et la Tarentaise est de celles-là.

Cette race, intelligemment sélectionnée, présente des sujets morphologiquement et fonctionnellement très améliorés par rapport à la souche primitive; mais, dans l'ensemble, les animaux sont restés extrêmement plastiques et rustiques. Ces qualités sont originelles, mais elles s'expliquent aussi par le mode d'exploitation auquel cst soumis' le bétail tarentais.

La stabulation pendant six mois de l'année, avec fourrages secs et le séjour en montagne pendant la belle saison, où les écarts de température peuvent dépasser $30^{\circ}$ au cours de la même journée, maintiennent la supériorité de la Tarentaise sur les races de plaine à climat régulier, quant à la résistance et aux facultés d'adaptation.

Elle a fait ses preuves dans des pays fort différents du sien, en particulier au Maroc; partout où les conditions extraordinairement sévères avaient fait abandonner les animaux d'autres races; elle a donné en croisement des sujets remarquables, doués de qualités tout à fait comparables aux sujets purs, mais parfaitement adaptés aux conditions de climat et de milieu.

La colonisation du territoire de Taza s'étend le long d'une étroite vallée, pointe terminale du Maroc fécond, enchassée entre le Riff et le Mioyen Atlas, couloir soumis aux vents d'Est desséchants et torrides de l'été, aux vents immodérẹes d'Ouest pendant l'hiver. Le sol de cette région du Maroc, quoique couvert d'alluvions dans sa partie basse, est moyennement riche : ce lambeau de l'Empire dit « fortune » reflète assez bien les caractéristiques d'une région Nord-Africaine très exposée à des conditions climatiques sévères, à des possibilités de végétation très mesurées mais à cause de celà très vite développées au maximum par les pionniers qui s'y installèrent après la pacification.

Importée dans cette région depuis plus de quinze ans, la race bovine tarenlaise a surtout contribué, par les succès de son métissage avec la race autochtone, à la création de sujets laitiers remarquables; le nombre des vaches pures reste insignifiant par rapport à celui des croisées. Cette dernière formule, malgré la rigueur du climat, a confirmé ses aptitudes à la production du lait. Le taureau tarentais demeure un raceur exceptionnel. Pour apprécier à sa juste valeur le mérite des métisses tarentaises, il était nécessaire de' souligner les conditions sévères imposées par 'le milieu.

Le régime saisonnier compte deux périodes : la période sèche de Mai à Septembre et la période des pluies d'Octobre à Avril. La température à l'ombre varie dans l'année de $-5^{\circ}$ à plus de $48^{\circ}$. L'état hygrométrique; suivant la saison, va de 25 en saison sèche à 90 en raison de pluies. L'eau existe partout, mais en saison sèche, le débit du fleuve principal se trouve réduit, rendant toutefois possible l'irrigation de quelques hectares dans chaque exploitation.

La végétation est naturellement assez abondante une partie de l'année; les pluies suffisent aux cultures fourragères : d'Octobre à Mai : bersim, orge fourrager; de Juin à Septembre, sont utilisés les chaumes, les productions fourragères en irrigation; 
luzerne, mais fourrager. Peu à peu, toutes les fermes reçoivent des installations de petite hydraulique destinées à réaliser ce que nous avons appelé « le cycle du vert "; il est intéressant de constater que la formation des remarquables troupeaux de métisses tarentaises a toujours précédé cette phase, laquelle est cependant rendue nécessaire pour permettre une alimentation plus favorable à la production laitière.

La race indigène marocaine, branche de la race brune d'Afrique du Nord, appartient au type ibérique; la lenteur des déplacements a éliminé - du moins avant l'utilisation intensive des transports maritimes - l'influence des sangs étrangers. Il est pourtant une exception qui aboutit à la création d'une véritable race indigène Noir-Pie dans les régions de Meknès et de Fès. Depuis celle-ci et bien postérieurement à elle, la colonisation du Maroc, admit dans ses étables et somme toute mêlait au sang des bovins indigènes des sangs de France ou des pays étrangers. Quelle a été jusqu'à présent, l'influence de ces courants nouveaux? Pratiquement nulle en ce qui concerne l'indigénat.

La belle vache marocaine mesure $1 \mathrm{~m} .25$ au garrot; elle peut atteindre 300 kilos. Le devant est ouvert, l'arrière-rnain présente les défauts communs aux autres espèces domestiques autochtones croupe en pupitre, bassin serré, jarret haut, défaut de musculature. Le profil est rectiligne, les cornes relevées, arquées blanches à la base, noires à leur extrémité, les segments osseux trapus, la côte ronde; le poil est fauve clair, châtain ou brun, foncé, les extrémités claires ou foncées s'accordent avec la robe. Bien nourrie, cette vache donne 5 à 8 litres de lait par jour, 500 à 1.000 litres par an selon ses origines, et exceptionnellement, certains sujets donnent 1.300 litres.

De nombreuses sous-races ont eté dénommées selon leur origine : celle d'Oulmès (la plus célèbre), de Khémisset, du Sebou, des Branes, et il y en a bien d'autres. Elles ne font, à travers les différences qu'elles présentent et qui traduisent les différences du sol, du climat et de nourriture, que signifier l'adaptation patiente de l'espèce à un certain cadre imposé par les conditions de vie.

La vache d'Oulmès, sélectionnée, bien nourrie, bien abrité, donne, pour un poids moyen de 250 kilos, 1.100 litres de lait en un peu plus de 200 jours de lactation; la moyenne est donc de 5 litres 4 par jour. Cette sous-race retire ses qualités des excellents fourrages des plateaux d'Oulmès.

Il n'est pas d'éleveur, si peu favorisées que soient certaines zones de sa région, qui n'ait constaté l'existence de vaches marocaines bien dessinées et prédestinées à faire des laitières. Ce sont des spécimens rares, il faut bien le dire, qu'il convient de rechercher, soit pour la sólection, 'soit pour servir à l'obtention de métis à $50 \%$ et $75 \%$.

La métisse tarentaise résiste bien aux vents chauds de l'été et accepte aux bonnes heures de la journée. le pacage sur chaumes. On a écrit que la Tarentaise est une formule mixte; en sous-entendant par là qu'elle a peu de qualités laitières; or, il faut l'évaluer au regard des conditions exceptionnellement peu favorables dans lesquelles les éleveurs sont obligés de la placer.

Heureux quant à son principe, ce métissage reste le meilleur à appliquer lorsque la laiterie a de modestes ressources alimentaires. Les croisées tarentaises s'entretiennent plus facilement que les marocaines et sont plus petites que leurs sceurs aînées, les métisses brunes de Suisse.

Le Tarentais s'allie parfaitement avec' la souche indigène; il donne des produits d'une belle robe froment à tête caractéristique : oreilles' larges et courtes, cornes fines bien dirigées vers l'avant, bout du nez court (on a pu dire carré) très noir; le dessus est horizontal, la tête ronde, le pis assez développé et bien dessiné, l'arrière-main très corrigée.

Bien nourrie, ou pour mieux dire, nourrie dans les meilleures conditions possibles, alors que les conditions restent défavorables, - à Taza, par exemple - les sujets donnent 1.850 litres de lait par an lorsqu'ils sont à $50 \%$ (14 litres au début et 5 litres au $11^{\mathbf{e}}$ mois), et 2.000 litres lorsqu'ils sont à $75 \%$ (16 litres au début et 5 litres au. $11^{\text {e }}$ mois).

Une laiterie de 30 vaches à $75 \%$, sise à Taza, disposant d'une surface irrigable de 3 hectares, fournit sous contrôle officiel plus de 72.000 litres de lait par an.

Alimentée en sec pendant l'été, donc sans irrigation, avec, pendant cette période, de rares ressources en vert, la croisée tarentaise à $50 \%$ donne 11 litres au début de la lactation et 3 litres au 11 mois, soit 1.500 litres par an; si elle est à $75 \%$, elle donnera 12 litres lorsqu'elle est fraiche et 4 litres au $11^{\circ}$ mois, soit 1.600 litres par an.

La durée de la lactation est très longue; elle dure d'une mise bas à l'autre, si l'on ne prend pas la précaution d'arrêter la traite un mois avant l'accouchement. Le modèle à $75 \%$ est un peu moins lourd que celui à $50 \%$; il est, comme nous 'venons de l'exprimer, plus laitier.

Les métisses tarentaises pèsent de 350 à 450 kilos; le tour de poitrine est de $1 \mathrm{~m} .80$ à 2 mètres; la taille, de $1 \mathrm{~m} .25$ à $1 \mathrm{~m} .40$.

Le croisement direct à $50 \%$ à partir d'une bonne femelle du pays et d'un mâle importé bien choisi réalise la formule de production la plus sûre et la plus économique. Il est contre-indiqué de poursuivre au delà le métissage, sauf lorsqu'on se propose une production laitiere; dans ce cas, on devra 
atteindre et s'arrêter au $75 \%$ de sang importé.

En corollaire, il s'ensuit que les animaux de boucherie et de travail doivent relever du croisement à $50 \%$.

L'histoire de l'acclimatement de nos races bovines laitières dans les pays exotiques n'est pas riche de réussites, Aussi tenons-nous à insister sur le destin des croisements de la race tarentaise dans une contrée où certaines conditions demeurent sévères.

La situation sanitaire dans le territoire de Taza n'est pas privilégiée; les charbons ne sont annihilés que grâce à des vaccinations annucllos; los piroplasmoses ont fait disparaître, avec la tuberculose et la brucellose, toutes les pépinières de vaches de races importees. Par contre, le métissage tarentais permet de conserver 9 sur 10 animaux jusqu'à l'âge de la réforme; notamment les atteintes de piroplasmoses sont beaucoup moins graves, le pronostic est toujours favorable quand l'éleveur fait intervenir le téchnicien au début de l'affection.

On peut visiter dans cette région :

'10 Des taureaux tarentais importés ou nés au Maroc dans des contrées plus favorisées, en excellent état de santé et d'entretien, conservant une activité génésique parfaite.

$2^{\circ}$ De's vaches tarentaises-marocaines à $50 \%$, chez lesquelles les' qualitès laitières 'sont déjà fort intéressantes, avec une lactation de 330 jours, un rendement moyen de 5,16 par jour et de 1.850 litres par an pour un poids de 430 kilos. Le rendement en lait pour 100 kilos vif est de 450 litres avec un pourcentage de 4,4 en matière grasse.

$3^{\circ}$ Des vaches tarentaises-marocaines à $75 \%$, plús harmonieuses, bien conformées et chez lesquelles des modifications morphologiques et fonctionnelle's dans le sens du Tarentais sont plus accentuées que dans le $50 \%$. La durée de lactation reste de 330 jours; elle se poursuit d'une mise bas à l'autre si l'on n'y prend garde; le rendement moyen' par jour est de 6 litres de lait, par an de 2.000 litres. Le poids moyen vif est de 400 kilos, donc avec un rendement, pour 100 kilos vifs, de 500 litres d'un lait dosant 4,4\% de matière grasse.

Un sujet $3 / 4$ de sang tarentais, d'un des meilleurs élevages, transplanté à Fès et nourri intensivement, a produit dans l'année 3.200 litres de lait.
1945 OKANER (H.). - Recherches sur les qualités morphologiques et physiques de la laine de la chèvre Angora. - Yüksek Zir, Enstitüsü Çalismalarindan (Ankara), no 148,

A l'inverse de ce qui existe chez le mouton, la laine de la chèvre angora diminue de longueur et augmente de finesse de l'avant vers l'arrière. Avec l'âge et particulièrement entre la $1^{\mathrm{r}} \mathrm{e}$ et la $2^{\mathrm{e}}$ année, elle devient moins uniforme, plus grossière et plus courte. L'extensibilité et l'élasticité, plus grande que dans la laine de mouton, augmentent avec l'âge et avec l'épaisseur de la fibre.

1946 HOWE (J.-W.). - Les effets de divers degrés de sang zébu sur l'adaptation du bétail laitier aux conditions de la Jamaïque. - Thèse du Iowa State Collège, $91 \mathrm{p}$.

Etude concernant l'influence du sang zébu sur trois races laitières importées en région tropicale : Jersey, Guernesey, Holstein. Les statistiques portent sur 1.187 vaches de la ferme gouvernementale de Hope (Jamaïque).

Les demi-sang donnent environ 500 livres de lait, au cours d'une lactạtion; les chiffres sont les suivants :

$\begin{array}{ccc}\text { Jersey } & \text { I/2 sang Jersey } & \text { Guernesey } \\ 4020 \text { livres } & 4520 \text { livres } & 3545 \text { livres } \\ 1 / 2 \text { sang Guernesey } & \text { Holstein } & 1 / 2 \text { song Holstein } \\ 3993 \text { livres } & 5090 \text { livres } & 5506 \text { livres }\end{array}$

La teneur en matière grasse est plus forte chez les animaux croisés : les demi-sang Jersey donnent le chiffre de $5,89 \%$ soit $1 \%$ de plus que les vaches pures; les demi-sang Guernesey $5,27 \%$, soit $0,42 \%$ de plus que les pures, et les demi-sang Holstein $4,42 \%$, soit $1 \%$ de plus que les $7 / 8$.

D'autre part, les demi-sang croissent plus vite en poids après deux ans jusqu'à l'âge de six ans. A cet âge, les demi-sang pèsent en moyenne 179 livres de plus que les pures. La faveur doit aller aux demisang Jersey.

1947 LALL (H.-K.). - Position du bétail dans l'Union indienne.- Ind. Farming. 8, 444.

Comparaison des cheptels de l'Union indienne et du Pakistan. Il y a plus de bovins dans l'Union; la plupart des races bonnes laitières, Sahiwal et Sindi, sont dans le Pakistan, mais dans le Sud de l'Union il y a aussi de bonnes races : Gir et Gaolao, buffle de race Murrah. La consommation du lait est plus forte dans le Pakistan : 200 grammes par jour au lieu de 165' dans l'Union.

L'Union possède beaucoup plus de chèvres :: 30.500 .000 contre 8.500 .000 . Alors que le Pakistan produit 6.300 .000 livres de laine « fine » avec une population ovine de 6 millions environ, l'Union indienne ne produit que 6.000 .000 de livres de laine avec 22 millions et demi de moutons. La production de la laine à tapis est de 18 millions de livres dans 